\title{
CARBONO ORGÁNICO DEL SUELO EN BOSQUES RIPARIOS, ARROZALES Y PASTURAS EN PIEDRAS, TOLIMA, COLOMBIA ${ }^{1}$
}

\author{
Hernán Jair Andrade-Castañeda², Milena Andrea Segura-Madrigal ${ }^{3}$,Andrés Sebastián Rojas-Patiño
}

\section{RESUMEN}

Carbono orgánico del suelo en bosques riparios, arrozales y pasturas en Piedras, Tolima, Colombia. El objetivo del estudio fue estimar el almacenamiento de carbono orgánico del suelo (COS) en la interface entre bosques riparios y una matriz de arrozales y pasturas con manejo orgánico. La investigación se efectuó en el municipio de Piedras, Departamento del Tolima, Colombia. Se seleccionaron dos lotes en producción (arroz y pastura) y se estimó el COS en ellos y en borde e interior de bosques riparios adyacentes a $0-20 \mathrm{~cm}$ de profundidad. Se cuantificó la densidad aparente y la concentración de carbono orgánico entre mayo y julio de 2013. Se estimó el potencial cambio en el COS por cambios de uso del suelo entre el arroz, pastura y los bosques riparios. Las interfaces arroz-bosque ripario y pastura-bosque ripario almacenaron en promedio 65,6 y $61,3 \mathrm{t} \mathrm{C} / \mathrm{ha}$, respectivamente, sin diferencias significativas $(p>0,05)$. No se detectaron diferencias estadísticas $(p>0,05)$ entre matrices agropecuarias (arroz y pastura) en ninguna de las variables. La posición de muestreo (matriz y borde e interior de los bosques) tuvo impacto significativo $(p>0,05)$ solo en la densidad aparente: $1,7 \mathrm{vs} 1,1 \mathrm{vs} 1,0 \mathrm{~g} / \mathrm{cm}^{3}$ al interior y el borde de los bosques y la matriz, respectivamente. El COS no fue afectado estadísticamente $(\mathrm{p}>0,05)$ por la posición en la interface bosque ripario-matriz. La conversión de bosques riparios a arrozales o pasturas con manejo orgánico no está emitiendo gases de efecto invernadero, por el contrario, está incrementando el COS en 3,2 t C/ha.

Palabras claves: cambios en el uso del suelo, gases de efecto invernadero, sumideros de carbono.

\begin{abstract}
Soil organic carbon in riparian forests, rice fields, and pastures in Piedras, Tolima, Colombia. The aim of the study was to estimate the soil organic carbon (SOC) storage in the interface between riparian forests and a matrix of rice fields and pastures with organic management. The study took place in Piedras, Tolima, Colombia. Two plots in production (rice and pasture) were selected and SOC was estimated in these areas and in the edge and the interior of adjacent riparian forests at a depth of 0 to $20 \mathrm{~cm}$. Bulk density and SOC concentration were quantified between May and July, 2013. Potential change in SOC storage due to land use change among rice fields, pastures, and riparian forests was estimated. The interfaces rice field-riparian forest and pasture-riparian forest stored an average of 65.6 and $61.3 \mathrm{t}$ $\mathrm{C} / \mathrm{ha}$, respectively, with no statistical differences $(\mathrm{p}>0.05)$. Statistical differences were not detected $(p>0.05)$ between agricultural matrices (rice fields and pastures) in any of the variables. The sampling position (matrix and the edge and interior of forests) had a significant impact $(\mathrm{p}<0.05)$ just in bulk density: 1.7 vs $1.1 \mathrm{vs} 1.0 \mathrm{~g} / \mathrm{cm}^{3}$ in interior and edge of the riparian forests and the matrix, respectively. SOC was not statistically affected ( $>0.05)$ by the position in the riparian forest-matrix interface. Conversion from riparian forests to rice fields or pastures with organic management is not emitting greenhouse gases, on the contrary, it is increasing SOC in $3.2 \mathrm{t} \mathrm{C/ha}$.
\end{abstract}

Keywords: land use changes, greenhouse gases, carbon sinks.

1 Recibido: 9 de julio, 2015. Aceptado: 10 de noviembre, 2015. Producto del proyecto de investigación "Oferta de Servicios Ambientales en Sistema de Uso del Suelo en la Zona Seca del Norte del Tolima", financiado por el Comité Central de Investigaciones de la Universidad del Tolima, Ibagué, Colombia.

2 Universidad del Tolima, Facultad de Ingeniería Agronómica, Grupo de Investigación Producción Ecoamigable de Cultivos Tropicales (PROECUT). Tel: (57) 2771212 ext. 9265, 9251, 9265. Barrio Santa Helena Parte Alta, Ibagué, Colombia. hjandrade@ut.edu.co, andres011191@hotmail.com

3 Universidad del Tolima, Facultad de Ingeniería Forestal, Grupo de Investigación Producción Ecoamigable de Cultivos Tropicales (PROECUT). Tel: (+57) 2771212 ext. 9251. Barrio Santa Helena Parte Alta, Ibagué, Colombia. masegura@ut.edu.co 


\section{INTRODUCCIÓN}

Una de las estrategias para la mitigación del cambio climático es la captura de carbono orgánico en el suelo (COS), principalmente en sistemas de uso de la tierra con componentes forestales (Alvarado et al., 2013). La deforestación ha causado la emisión de grandes cantidades de gases de efecto invernadero (GEI), que corresponden a un 6 a $17 \%$ de las emisiones antropogénicas de $\mathrm{CO}_{2}$ a la atmósfera. Entre los años 2000 y 2010 , se emitieron aproximadamente $1,0 \mathrm{Pg}^{3} \mathrm{C} /$ año por destrucción del bosque tropical y uso de la tierra (Baccini et al., 2012).

El uso del suelo desempeña un papel importante en la mitigación del cambio climático, pues este es el mayor sumidero de carbono (FAO, 2002a). El cambio en el uso del suelo causa emisiones netas de GEI (IPCC, 2013). Por ejemplo, conversiones de cultivos de arroz paddy a hortalizas contribuyeron a disminuir el almacenamiento de carbono orgánico del suelo (COS) en China (Wang et al., 2014). En Argentina, el abandono de arrozales redujo inicialmente el COS en los primeros cuatro años, pero luego se incrementó levemente hasta retornar a los niveles iniciales a los quince años (Desjardins et al., 2006). En suelos de páramo en Colombia, Andrade et al. (2014) encontraron que el pastoreo extensivo favorece la acumulación de COS en las capas superficiales del suelo $(0-30 \mathrm{~cm})$. El manejo de ganado ha cambiado el suministro de servicios ecosistémicos, tal como la captura de carbono, en diferentes formas (Van Oudenhoven et al. 2012). Otra variable determinante para la acumulación de COS es la pendiente, pues Mosquera et al. (2012) encontraron mayor contenido de COS en suelos planos que en aquellos con pendiente cuando estos cambiaron su uso. Por esta razón, se deben aplicar métodos que secuestren carbono en los suelos o en la biomasa (Naciones Unidas, 1998).

Se han reportado 63,7 millones de hectáreas de bosque en todo el territorio nacional colombiano, de ellas solo 3,9 millones corresponden a bosques riparios (FAO, 2002b). Se estimó una disminución de 3,3 millones ha de bosques en Colombia entre 2002 y 2010 , de los cuales el $1 \%$ son bosques riparios (FAO, 2010). Estos cambios implican una reducción del COS en el trópico de aproximadamente $75 \%$ (Lal, 2004). Estas cifras son alarmantes, ya que

${ }^{3} 1 \mathrm{Pg}=10^{9} \mathrm{t}=10^{15} \mathrm{~g}$ los bosques son protectores de los recursos suelo, agua, fauna, flora e interventores directos en las producciones agropecuarias. Se conoce que sin agua no existe ninguna producción, de ahí que el manejo y conservación de bosques se debe hacer de manera integral (Arcos, 2005).

Los efectos de conversión de tierras en el COS han sido conducidos principalmente en zonas húmedas y no en secas (Jiménez et al., 2011). En concordancia con esto, el objetivo del estudio fue estimar el almacenamiento del COS en la interface entre bosques riparios y una matriz de arrozales y pasturas con manejo orgánico.

\section{MATERIALES Y MÉTODOS}

\section{Área de estudio}

El estudio se realizó en la Hacienda Gascoña en el municipio de Piedras, departamento del Tolima, Colombia, ubicada a $4^{\circ} 24^{\prime} 17,9^{\prime \prime}$ - $4^{\circ} 24^{\prime} 24,7^{\prime \prime} \mathrm{N}$ y $75^{\circ} 02^{\prime} 46,0^{\prime \prime}$ - 7502'08,0” O entre los meses de mayo y julio del 2013. La estimación del COS fue realizada una vez durante este período. El sitio se encuentra en la zona de vida de bosque seco tropical (Holdridge, 1996), con una altitud de $403 \mathrm{msnm}$ y una temperatura promedio de $26{ }^{\circ} \mathrm{C}$ (Alcaldía de Piedras -Tolima, 2014). El área de estudio presenta una precipitación promedio anual que varía de 1300 a $1600 \mathrm{~mm}$ (Corporación Autónoma Regional del Tolima, 2009), donde el mayor periodo de lluvias ocurre en la mitad del primero y segundo semestre del año (Alcaldía Municipal de Piedras, 2008). La Hacienda Gascoña se encuentra en el abanico de Ibagué, que cubre una superficie aproximada de 700 $\mathrm{km}^{2}$ y es una serie de depósitos fluviovolcánicos, que están constituidos por flujos de lodo y escombros, depósitos de tamiz y llanura de inundación, y flujos piroclásticos derivados principalmente por la actividad del Nevado del Tolima. Los suelos pertenecen a la unidad VWA (Typic Haplustepts), que se caracterizan por ser poco evolucionados, bien drenados y profundos a moderadamente profundos (IGAC, 2004).

En el municipio de Piedras se emplean varios sistemas de uso del suelo, los cultivos más representativos en el sector agrícola son el arroz, maíz, sorgo y yuca. La producción pecuaria está representada por ganado doble propósito y producción porcina (Alcaldía Municipal de Piedras, 2008). Este 
municipio cuenta con un área de 123,15 ha de bosque protector, las cuales colindan con cauces hídricos y representan solo el $0,35 \%$ del área del municipio (Corporación Autónoma Regional del Tolima, 2009).

\section{Sistemas de uso del suelo a evaluar}

Se seleccionaron fragmentos ribereños de bosque seco tropical colindantes con una matriz de arrozales y pasturas en sistemas de manejo orgánico. En el manejo de estos sistemas de producción se prepara el suelo con cero labranza, realizando dos pases de rastra y la incorporación de 1 t/ha de compost. En el arroz se maneja la soca, es decir, se cosecha (plataforma, trilla y tolva) y luego se deja repoblar naturalmente el cultivo durante los dos siguientes ciclos. Cada tres ciclos de cultivo de arroz, se vuelve a preparar el suelo.
En cada interface matriz-bosque ripario, se trazaron cinco transectos, los que se consideraron como repeticiones, en donde se ubicaron cuatro parcelas temporales de muestreo: dos en el interior de los fragmentos de bosque, uno en el borde y uno en la matriz agropecuaria (Figura 1).

\section{Estimación del carbono orgánico del suelo}

El carbono orgánico del suelo (COS) se estimó a una profundidad de 0 a $20 \mathrm{~cm}$, según la metodología propuesta por MacDiken (1997):

$$
C O S=\% C O \times D A \times P S
$$

Donde:

COS: carbono orgánico en el suelo ( $\mathrm{t} / \mathrm{ha}$ ).

\%CO: porcentaje de carbono orgánico en el suelo.

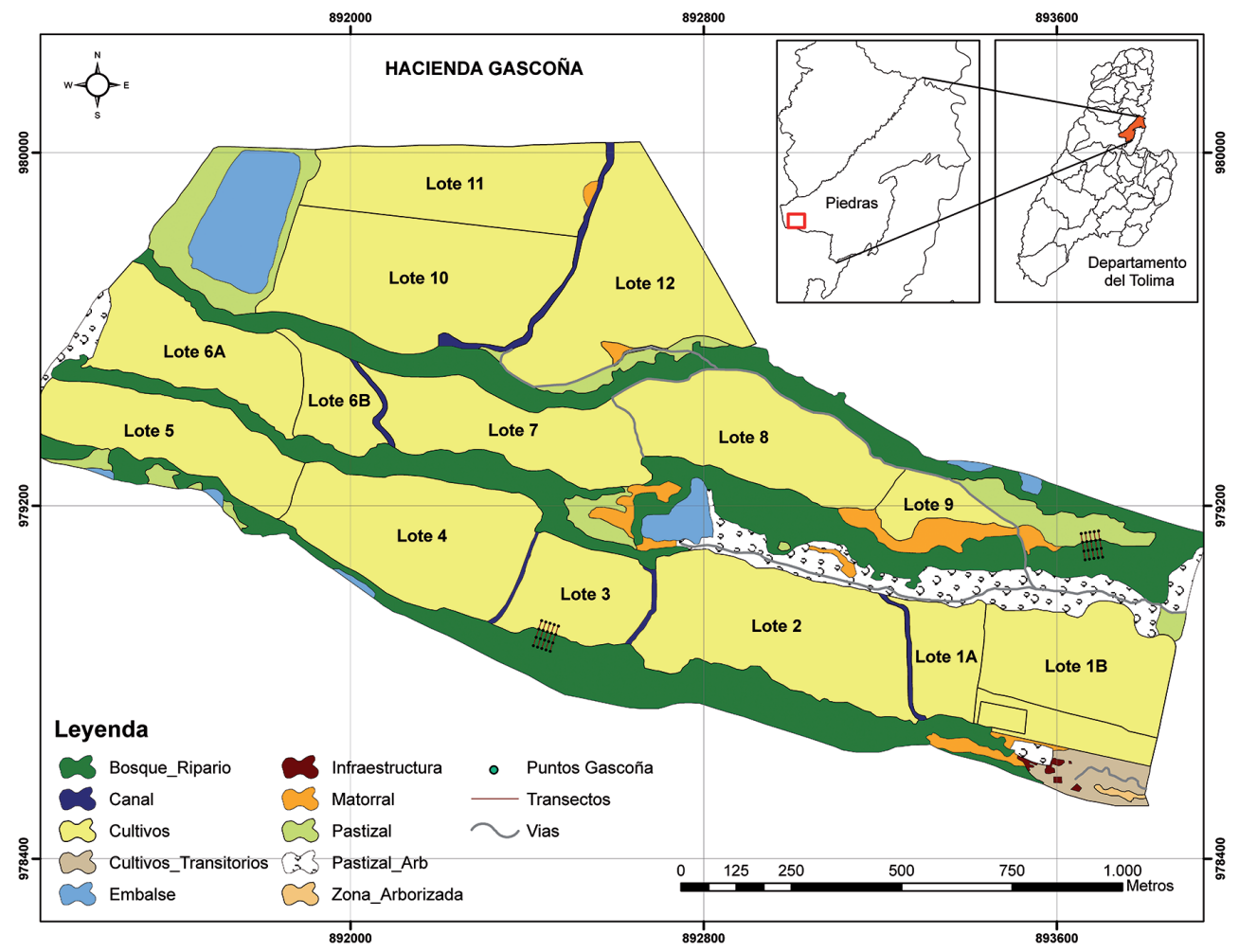

Figura 1. Ubicación de las parcelas de muestreo para estimar la interacción en el almacenamiento de carbono orgánico del suelo (COS) entre bosques riparios y una matriz de arrozales y pasturas con manejo orgánico, en la Hacienda Gascoña, Piedras, Tolima, Colombia.

Figure 1. Location of sampling plots to estimate the interaction of soil organic carbon (SOC) storage between riparian forests and a matrix of rice fields and pastures with organic management, in Hacienda Gascoña, Piedras, Tolima, Colombia. 
DA: densidad aparente $\left(\mathrm{g} / \mathrm{cm}^{3}\right)$.

Ps: profundidad del suelo $(\mathrm{cm})$.

No se descontó la pedregosidad en el cálculo del almacenamiento de carbono en suelos, ya que esta variable resultó despreciable al realizar las minicalicatas para estimar densidad aparente (DA) y concentración de COS.

El carbono orgánico almacenado en el suelo se calculó utilizando una base fija de densidad aparente, la cual fue el promedio de los relictos de bosque de las interfaces estudiadas, en lugar de una profundidad fija, esto debido a que cambios en el uso del suelo acfectan la DA (Ellert et al., 2002; Amézquita et al., 2008).

En cada parcela, se tomaron veinticinco submuestras de suelo (IPCC, 1996), de las cuales se extrajo una muestra compuesta de aproximadamente 250 g para estimar la concentración de carbono orgánico con el método de Walkley y Black (1934) en el laboratorio Laserex de la Universidad del Tolima. Estas muestras se secaron en horno $\left(105^{\circ} \mathrm{C}\right.$ hasta peso constante) y se pesaron. La DA se estimó empleando el método del cilindro de volumen conocido, que consiste en extraer una muestra con un cilindro de volumen conocido a la profundidad de estudio (Andrade e Ibrahim, 2003), resultando la DA el cociente entre el peso seco y el volumen interno del cilindro.

Impacto de potenciales cambios de uso del suelo en la dinámica del COS

Se simuló el comportamiento del COS a cambios de uso del suelo en las dos matrices agropecuarias: arrozal y pastura. Se empleó el COS de las matrices y de las partes internas de cada bosque ripario aledaños a la matriz correspondiente. Las estimaciones se hicieron con base en $\mathrm{t} \mathrm{CO}_{2}$, lo cual implica emisiones de este GEI o fijación neta de COS.

\section{Análisis estadísticos}

Se utilizó un diseño experimental completamente al azar con arreglo factorial (sistema por posición). Se probaron los tres supuestos para realizar un diseño experimental: tratamientos independientes, los errores se distribuyeron con media cero y varianza $\sigma^{2}$, y con homogeneidad de varianzas (Steel y Torrie, 1988). Se realizaron pruebas de comparación de medias de Fisher a los tratamientos simples y factoriales, se realizó un análisis de interacción para observar la relación de carbono, entre bloques y parcelas de diferentes bloques. Los análisis estadísticos fueron realizados con el programa InfoStat.

\section{RESULTADOS Y DISCUSIÓN}

\section{Densidad aparente del suelo}

La DA de los suelos en el interior de los bosques riparios fue estadísticamente mayor $(p<0,05)$ en ambas interfaces, que en los bordes y en la matriz agropecuaria, con valores de 1,7 vs 1,1 vs $1,0 \mathrm{~g} / \mathrm{cm}^{3}$, respectivamente (Figura 2a). La matriz agropecuaria estudiada (arrozal y pastura) tampoco afectó estadísticamente $(p>0,05)$ esta variable edáfica. No se detectó interacción estadística $(\mathrm{p}>0,05)$ entre sistemas y posiciones, es decir, se mantuvo la misma tendencia de la DA en las dos interfaces.

Los resultados de estudios anteriores no son consistentes en cuanto al impacto de sistemas de uso del suelo en la DA del suelo (Rhoades et al., 2004; Pinno y Wilson, 2011); sin embargo, es de esperarse que la actividad agropecuaria incremente la densidad aparente de los suelos (Zotarelli et al., 2005; Jiang et al., 2011). De la misma forma, Decaëns et al. (2002) afirmaron que las pasturas viejas incrementan la DA en comparación con pasturas recientes y cultivos en sabanas colombianas. En el estudio realizado ocurrió lo contrario, los remanentes de bosque presentaron la mayor DA que los bordes en los campos agropecuarios $\left(1,8\right.$ vs $\left.1,0 \mathrm{~g} / \mathrm{cm}^{3}\right)$, probablemente por el manejo orgánico de ambos sistemas (arroz y pasturas).

\section{Concentración de carbono orgánico del suelo}

La concentración de COS varió entre 1,5 y $2,6 \%$ en los $20 \mathrm{~cm}$ superficiales de estos suelos. No se detectaron diferencias estadísticas $(p>0,05)$ en la concentración de COS entre usos del suelo, siendo un 2,0 y $1,8 \%$ para arrozales y pasturas, respectivamente (Figura $2 b$ ). Se presentó una interacción estadística entre el sistema de uso del suelo-posición $(\mathrm{p}<0,05)$ en la concentración del COS; esto debido a que en la interface arrozal-bosque ripario, no se detectaron diferencias significativas $(\mathrm{p}>0,05)$ en esta variable, 


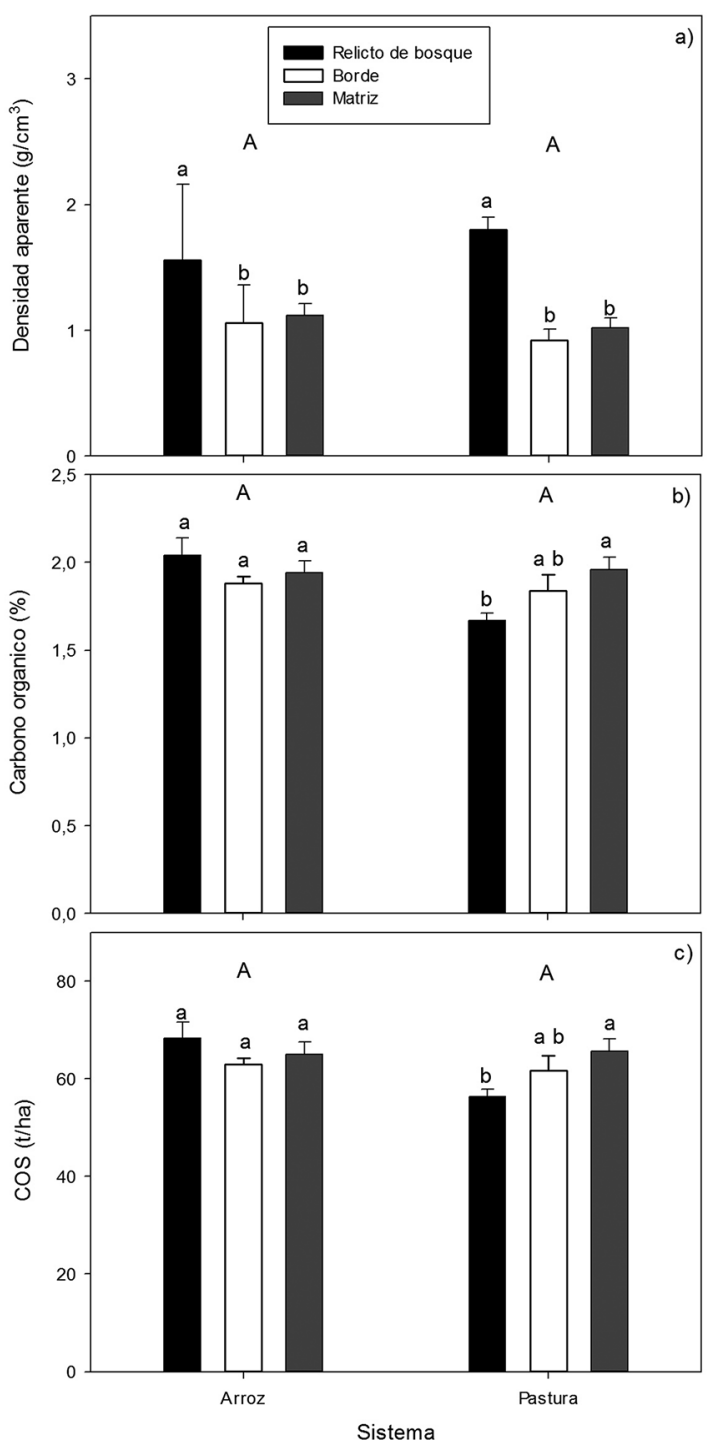

Figura 2. Densidad aparente (a), carbono orgánico (b) y almacenamiento de carbono orgánico del suelo (c) a profundidad de 0 a $20 \mathrm{~cm}$ en interfaces arrozbosque ripario y pasto-bosque ripario en Piedras, Tolima, Colombia. 2013.

Las barras de error corresponden al error estándar. Letras minúsculas y mayúsculas diferentes indican diferencias significativas $(\mathrm{p}<0,05)$ entre posiciones y sistemas, respectivamente.

Figure 2. Bulk density (a), organic carbon (b), and soil organic carbon storage (c) at a depth of 0 to $20 \mathrm{~cm}$ in interfaces rice fields-riparian forests and pasturesriparian forests in Piedras, Tolima, Colombia. 2013. Error bars correspond to standard error. Diferent lower and upper case letters indicate significant differences $(\mathrm{p}<0.05)$ between positions and systems, respectively. contrastando con la mayor concentración de COS en las pasturas que en el interior de los bosques riparios $(2,0 \pm 0,2$ vs $1,7 \pm 0,1 \%$, respectivamente; Figura $2 b)$.

\section{Almacenamiento de carbono orgánico de suelos}

Las dos matrices no presentaron diferencias significativas $(p>0,05)$ en cuanto a su almacenamiento de COS; en la profundidad estudiada. La alta variabilidad encontrada dentro de cada uso del suelo, tal como los hallazgos de este estudio, indican el potencial para mejoramientos en sitios con pobre almacenamiento de carbono; mientras que las interacciones sugieren que algunos cambios de uso del suelo pueden contribuir a mitigar el cambio climático al acumular más COS (Lavelle et al., 2014).

Las interfaces arroz-bosque ripario y pastobosque ripario almacenaron un contenido de $\mathrm{COS}$ estadísticamente similar ( $p>0,05$ ): 65,6 y 61,3 t C/ha, respectivamente. De la misma forma, la posición de muestreo no afectó estadísticamente $(\mathrm{p}>0,05)$ el COS almacenado (Figura 2c). Estos hallazgos contrastan con lo reportado en un estudio donde se afirmó que los bosques de galería en un paisaje agrícola del Cerrado en Brasil almacenaron más carbono en los 60 $\mathrm{cm}$ superficiales de suelo que los otros ecosistemas, incluyendo pasturas y humedales (Wantzen et al., 2012).

El carbono almacenado $(50,2$ a 87,1 t/ha) resultó muy alto en comparación con lo reportado por Batjes (1999) con los datos de FAO-IIASA en zonas agroecológicas y climáticas similares del trópico (36 a $38 \mathrm{t} / \mathrm{ha}$ ). Esto confirma la influencia de otros factores formadores de suelo y de manejo que determinan la dinámica del COS (FAO, 2002a). El mantenimiento del COS en el uso arrocero se puede deber a la realización de buenas prácticas agrícolas, como la incorporación de residuos de cosecha (Carvajal et al., 2009) y la labranza de conservación (Oldeman et al., 1991). El contenido de COS asociado con los macroagregados estables al agua fue mayor que el asociado con microagregados (Wang et al., 2014); los primeros son menores en suelos agrícolas (Zotarelli et al., 2005; Jiang et al., 2011).

\section{Impacto de potenciales cambios de uso del suelo en la dinámica del COS}

Se encontraron resultados contrastantes en el efecto del cambio de uso del suelo entre la matriz agropecuaria 
y los bosques riparios. Los mayores cambios en el almacenamiento de COS se manifiestan cuando se convierten áreas de pastura a bosque o viceversa. Si las pasturas son abandonadas y se propicia la regeneración natural de los bosques, es posible reducir el COS en un promedio de 35,8 t CO/ha (Cuadro 1), causando una emisión neta de este GEI, el cambio contrario causa una fijación neta de COS. En contraste, al eliminar los bosques riparios y establecer arrozales, el COS tiende a disminuir en 12,3 $\mathrm{t} \mathrm{CO}_{2}$ /ha (Cuadro 1).

En el caso de las pasturas era de esperarse ese incremento en el COS, ya que la dinámica de las raíces finas por efecto de su senescencia por defoliación a causa del pastoreo, hace que grandes cantidades de carbono se estén incorporando al suelo (Andrade et al., 2008; Andrade et al., 2014). Este carbono puede acumularse en capas más profundas del suelo con menor susceptibilidad de descomposición (Fearnside y Barbosa, 1998). La tendencia de reducción de DA con incrementos de concentración de COS coincide con lo manifestado en otros estudios (Alvarado et al., 2013), ya que la materia orgánica presenta una alta organización estructural (Perie y Ouimet, 2007).

Cuadro 1. Impacto del cambio de uso del suelo en el almacenamiento de $\mathrm{COS}$ (t $\mathrm{CO}_{2} / \mathrm{ha}$ ) a una profundidad de 0 a $20 \mathrm{~cm}$ de profundidad en Hacienda Gascoña, Doima, Tolima, Colombia. 2013.

Table 1. Impact of land use change in SOC storage (t CO2/ ha) at a depth of 0 to $20 \mathrm{~cm}$ in Hacienda Gascoña, Doima, Tolima, Colombia. 2013.

\begin{tabular}{|c|c|c|c|}
\hline & \multicolumn{3}{|c|}{ Pasturas } \\
\hline & & \multicolumn{2}{|c|}{ Uso futuro } \\
\hline & & Bosque ripario & Pastura \\
\hline \multirow[t]{5}{*}{ Uso actual } & Bosque ripario & 0 & 35,8 \\
\hline & Pastura & $-35,8$ & 0 \\
\hline & & \multicolumn{2}{|l|}{ Arrozales } \\
\hline & & \multicolumn{2}{|c|}{ Uso futuro } \\
\hline & & Bosque ripario & Arroz \\
\hline \multirow[t]{2}{*}{ Uso actual } & Bosque ripario & 0 & $-12,3$ \\
\hline & Arroz & 12,3 & 0 \\
\hline
\end{tabular}

Valores negativos (en cursiva): indican reducción en el almacenamiento de COS, mientras que valores positivos señalan: incrementos / Negative values (in italic): indicate a reduction in the SOC storage. Positive values show increments.
En este estudio se consideró el suelo como sumidero de carbono, pero si se consideran otros sumideros, como la biomasa de árboles (Andrade et al., 2014), los bosques riparios conservan más carbono y proveen otros servicios ecosistémicos (FAO, 2010). Estos bosques están ubicados en las zonas aledañas a las corrientes de agua, y desempeñan un papel importante en la preservación del recurso hídrico y estabilización de los cauces, como corredores de dispersión de la biota y como albergues para la fauna en épocas secas (FAO, 2002b).

Relación entre la densidad aparente (DA) y la
concentración de carbono orgánico del suelo (COS)

En términos generales, no se encontró una relación estrecha entre la concentración de COS y la DA en este paisaje agropecuario $(r=-0,09 ; \mathrm{p}=0,56)$ (Figura $3 \mathrm{c})$. Se encontraron tendencias contrastantes en la relación entre estas dos variables en los $20 \mathrm{~cm}$ de profundidad del suelo en las dos interfaces analizadas. En el caso de las matrices agropecuarias (arrozal y pastura), la DA aumentó con incrementos en la concentración del $\operatorname{COS}(r=0,40 ; p=0,08)$ (Figura 3a); mientras que en los bosques riparios, la DA disminuyó con aumentos del $\operatorname{COS}(r=-0,15 ; p=0,54)$ (Figura 3b).

$\mathrm{El}$ aumento de la DA al incrementarse el porcentaje de $\mathrm{COS}$ en las matrices estudiadas es controversial. Andrade et al. (2014) encontraron tendencias opuestas, con relaciones directas en bosques e inversas en pasturas en uso. Este resultado puede deberse a que no se realizan labores que reestructuren los macro agregados y produzcan el mullimiento del suelo y a que el paso continúo de maquinaria y ganado causen compactación, por lo cual el COS a los 20 $\mathrm{cm}$ sea húmico y la fracción lábil se encuentre en zonas superficiales (Fassbender y Bornemisza, 1994; Martínez et al., 2008). En los relictos de bosque y en todo el sistema evaluado la DA se reduce al incrementarse el porcentaje de COS, mejorando la calidad del suelo, al incrementarse el contenido de macroagregados y consecuentemente de los flujos de agua y nutrimentos (Fassbender, 1993; Fassbender y Bornemisza, 1994; Martínez et al., 2008). Resultados de esta relación inversa también fueron reportados por Alvarado et al. (2013) en suelos cafeteros de Colombia, con diferentes sistemas de producción. 

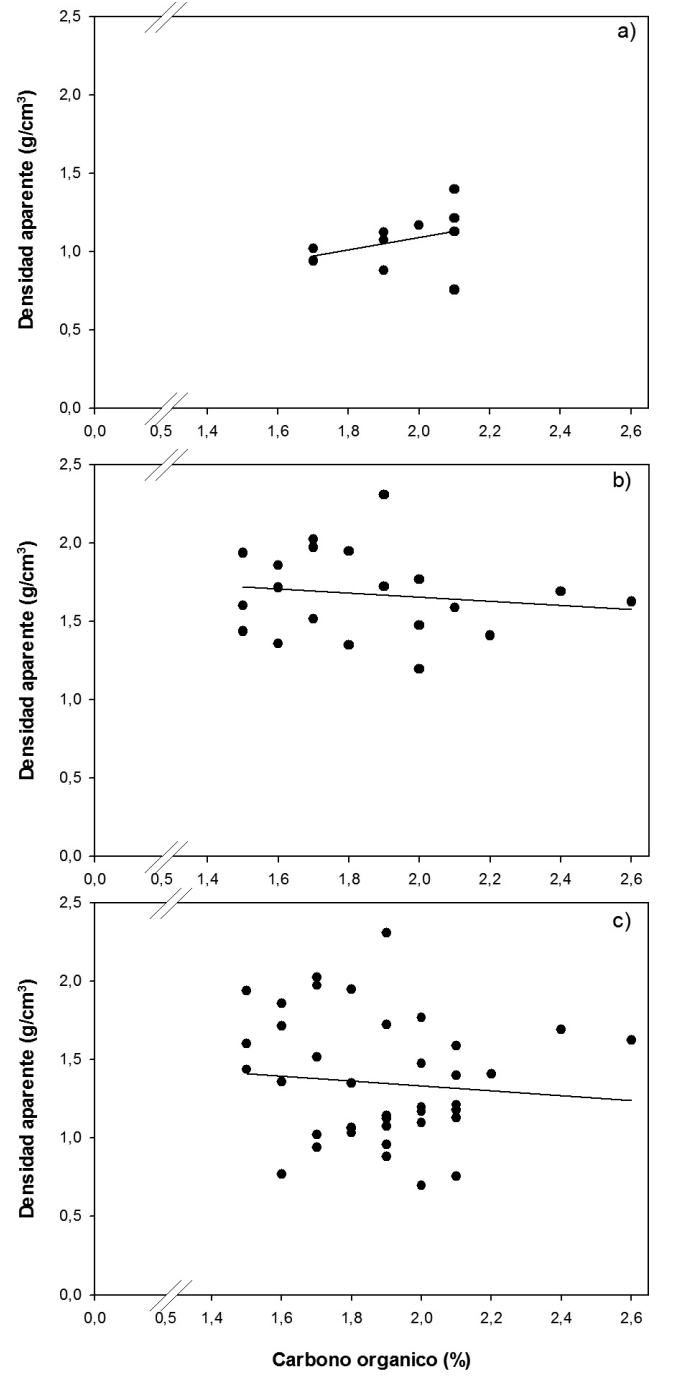

Figura 3. Relación entre la densidad aparente y el porcentaje de carbono orgánico en suelo de las matrices arroz-pasto (a), relación entre la densidad aparente y el porcentaje de carbono orgánico en suelo de los bosques riparios (b) relación entre la densidad aparente y el porcentaje de carbono orgánico en todo el sistema (c) a una profundidad de $20 \mathrm{~cm}$ en interfaces arroz-bosque ripario y pasto-bosque ripario en Piedras, Tolima, Colombia. 2013.

Figure 3. Relationship between bulk density (BD) and percentage of soil organic carbon (SOC) in the matrices rice fields pastures- (a), relationship between $\mathrm{BD}$ and percentage of SOC in riparian forests (b), and relationship between $\mathrm{BD}$ and percentage of SOC in the whole system (c) at a depth of $20 \mathrm{~cm}$ in interfaces rice fields-riparian forests and pastures-riparian forests in Piedras, Tolima, Colombia. 2013.

\section{AGRADECIMIENTOS}

Los autores desean expresar su gratitud al Comité de Investigaciones de la Universidad del Tolima (Ibagué, Colombia) por financiar el proyecto "Oferta de Servicios Ambientales en la Zona Seca del Norte del Tolima", código 450112.

\section{LITERATURA CITADA}

Alcaldía de Piedras - Tolima. 2014. Nuestro municipio: información general. http://www.piedras-tolima.gov. co/informacion_general.shtml (consultado 2 oct. 2014).

Alcaldía Municipal de Piedras. 2008. Plan de desarrollo Alcaldía Municipal de Piedras Tolima "gobierno de oportunidades para nuestra gente". Alcaldía Municipal de Piedras. Piedras, Tolima, COL.

Alvarado, J., H.J. Andrade, y M. Segura. 2013. Almacenamiento de carbono orgánico en suelos en sistemas de producción de café (Coffea arabica L.) en el municipio del Líbano, Tolima, Colombia. Col. For. 16:21-31.

Amézquita, M.C., P. Buurman, and M.A. Ibrahim. 2008. C stocks and sequestration. In: L. 'tMannetje et al., editors, Carbon sequestration in tropical grassland ecosystems. Wageningen Academic Publishers, HOL. p. 49-63.

Andrade, H., y M. Ibrahim. 2003. ¿Cómo monitorear el secuestro de carbono en los sistemas silvopastoriles? Agroforestería en las Américas 10:109-116.

Andrade, H.J., R. Brook, and M. Ibrahim. 2008. Growth, production and carbon sequestration of silvopastoral systems with native timber species in the dry lowlands of Costa Rica. Plant Soil 308:11-22.

Andrade, H.J., E.L. Espinoza, and H.A. Moreno. 2014. Impact of grazing on soil organic storage carbon in high lands of Anaime, Tolima, Colombia. Zootec. Trop. 32:7-21.

Arcos, I. 2005. Efecto del ancho de los ecosistemas riparios en la conservación de la calidad del agua y la biodiversidad en la microcuenca del río Sesesmiles, Copán, Honduras. Tesis MSc., CATIE, Turrialba, CRC.

Baccini, A., S.J. Goetz, W.S. Walker, N.T. Laporte, M. Sun, D. Sulla, J. Hackler, P.S.A. Beck, R. Dubayah, M.A. Friedl, S. Samanta., and R.A. Houghton. 2012. Estimated carbon dioxide emissions from tropical 
deforestation improved by carbon-density maps. Nat. Clim. Change 2:182-185.

Batjes, N.H. 1999. Management options for reducing $\mathrm{CO}_{2}-$ concentrations in the atmosphere by increasing carbon sequestration in the soil. ISRIC. Wageningen, HOL.

Carvajal, A.F., A. Feijoo, H.Y. Quintero, y M.A. Rondón. 2009. Carbono orgánico del suelo en diferentes usos del terreno de paisajes andinos colombianos. R. C. Suelo Nutr. Veg. 9:222-235.

Corporación Autónoma Regional del Tolima. 2009. Agenda ambiental del municipio de Piedras, Documento Técnico. 15. http://www.cortolima.gov.co/sites/ default/files/images/stories/centro_documentos/ estudios/agendas/2009_Agenda_Ambiental_del_ Municipio_de_Piedras.pdf (consultado 11 may. 2015).

Decaëns, T., N. Asakawa, J.H. Galvis, R.J. Thomas, and E. Amézquita. 2002. Surface activity of soil ecosystem engineers and soil structure in contrasted land use systems of Colombia. Eur. J. Soil Biol. 38:267-271.

Desjardins, T., P.J. Folgarait, A. Pando-Bahuon, C. Girardin, and P. Lavelle. 2006. Soil organic matter dynamics along a rice chronosequence in north-eastern Argentina: Evidence from natural ${ }^{13} \mathrm{C}$ abundance and particle size fractionation. Soil Biol. Biochem. 38:2753-2761.

Ellert, B.H., H.H. Janzen, and T. Entz. 2002. Assessment of a method to measure temporal change in soil $\mathrm{C}$ storage. SSSAJ 66:1687-1695.

FAO. 2002a. Captura de carbono en los suelos para un mejor manejo de la tierra. FAO, Roma, ITA.

FAO. 2002b. Estado de la información forestal en Colombia. FAO, Santiago, CHI.

FAO. 2010. Evaluación de los recursos forestales mundiales. FAO, Roma, ITA.

Fassbender, H., y E. Bornemisza. 1994. Química de suelos: con énfasis en suelos de América Latina. IICA. San José, CRC.

Fassbender, H. 1993. Ciclos de la materia orgánica. En Modelos edafológicos de sistemas agroforestales. $2^{\text {da }}$ ed. CATIE. Turrialba. CRC. p. 165-294.

Fearnside, P.M., and R.I. Barbosa. 1998. Soil carbon changes from conversion of forest to pasture in Brazilian Amazonia. For. Ecol. Manag. 108:147-166.

Holdridge, L. 1996. Ecología basada en zonas de vida. $4^{\mathrm{a}}$ reimpresión. IICA, San José, CRC.

IGAC (Instituto Geográfico Agustín Codazzi). 2004. Estudio general de suelos y zonificación de tierras. Departamento de Tolima, Bogotá DC, COL.
IPCC (Intergovernmental Panel on Climate Change). 1996. Report of the twelfth session of the intergovernmental panel on climate change. In: IPCC, editor, Revised 1996 guidelines for national greenhouse gas inventories. IPCC, MEX.

IPCC (Intergovernmental Panel on Climate Change). 2013. Cambio climático 2013: bases físicas. Contribución del grupo de trabajo I al quinto informe de evaluación del grupo intergubernamental de expertos sobre el cambio climático. IPCC, Ginebra, SUI.

Jiang, X., A.L. Wright, X. Wang, and F. Liang. 2011. Tillageinduced changes in fungal and bacterial biomass associated with soil aggregates: a long-term field study in a subtropical rice soil in China. Appl. Soil Ecol. 48:168-173.

Jiménez, J.J., K. Lorenz, and R. Lal. 2011. Organic carbon and nitrogen in soil particle-size aggregates under dry tropical forests from Guanacaste, Costa Rica - Implications for within-site soil organic carbon stabilization. Catena 86:178-191.

Lal, R. 2004. Soil carbon sequestration impacts of global climate change and food security. Sci. 304:1623-1627.

Lavelle, P., N. Rodríguez, O. Arguello, J. Bernal, C. Botero, P. Chavarro, Y. Gómez, A. Gutiérrez, M.P. Hurtado, S. Loaiza, S.X. Pulido, E. Rodríguez, C. Sanabria, E. Velásquez, and S.J. Fonte. 2014. Soil ecosystem services and land use in the rapidly changing Orinoco River Basin of Colombia. Agric. Ecosys. Environ. 185:106-117.

MacDiken, K. 1997. A guide to monitoring carbon storage in forestry and agroforestry projects. Winrock International, Arlington, VA, USA.

Martínez, E., J.P. Fuentes, y E. Acevedo. 2008. Carbono orgánico y propiedades del suelo. R.C. Suelo Nutr. Veg. 8:68-96

Mosquera, O., P. Buurman, B.L. Ramírez, and M.C. Amézquita. 2012. Carbon replacement and stability changes in short-term silvo-pastoral experiments in Colombian Amazonia. Geoderma 170:56-63.

Naciones Unidas. 1998. Protocolo de Kyoto de la convención marco de las Naciones Unidas sobre el cambio climático. Naciones Unidas, WA, USA.

Oldeman, L.R., R.T.A. Hakkeling, and W.G. Sombroek. 1991. World map of the status of human induced soil degradation: an explanatory note. 2nd ed. United Nation Environment Programme, Nairobi, KEN.

Perie, C., and R. Ouimet. 2007. Organic carbon, organic matter and bulk density relationships in boreal forest soils. Canadian J. Soil Sci. 38:315-325. 
Pinno, B., and S. Wilson. 2011. Ecosystem carbon changes with woody encroachment of grassland in the northern Great Plains. Ecoscience 18:157-163.

Rhoades, C., S. Miller, and M. Shea. 2004. Soil properties and soil nitrogen dynamics of prairie-like forest openings and surrounding forests in Kentucky's Knobs Region. Am. Midl. Nat. 152:1-11.

Steel, R.G., y J.H. Torrie. 1988. Bioestadística: principios y procedimientos. $2^{\text {a }}$ ed. McGraw-Hill, MEX.

Van Oudenhoven, A.P.E., K. Petz, R. Alkemade, L. Hein, and R.S. de Groot. 2012. Framework for systematic indicator selection to assess effects of land management on ecosystem services. Ecol. Indic. 21:110-122.

Walkley, A., and C.A. Black. 1934. An examination of the Degtajareff's method for determining soil organic matter and a proposed modification of the chromic acid titration method. Soil Sci. 37:29-38.

Wang, H., D. Guan, R. Zhang, Y. Chen, Y. Hu., and L. Xiao. 2014. Soil aggregates and organic carbon affected by the land use change from rice paddy to vegetable field. Ecol. Eng. 70:206-211.

Wantzen, K.M., E.G. Couto, E.E. Mund, R.S.S. Amorim, A. Siqueira, K. Tielbörger, and M. Seifan. 2012. Soil carbon stocks in stream-valley-ecosystems in the Brazilian Cerrado agroscape. Agric. Ecosys. Environ. 151:70-79.

Zotarelli, L., B. Alves, S. Urquiaga, E. Torres, H.P Dos Santos, K. Paustian, R.M. Boddey, and J. Seis. 2005. Impact of tillage and crop rotation on aggregateassociated carbon in two oxisols. Soil Sci. Am. J. 69:482-49. 